Revue des patrimoines

$37 \mid 2018$

Jardins collectifs : de l'abbé Lemire aux jardins d'insertion. Typologies - Expériences - Enjeux de conservation

\title{
Les jardins familiaux des Petits-Bois à Versailles : un patrimoine vivant à préserver
}

The 'Petits-Bois' family gardens at Versailles, a living heritage to be preserved

\section{Fabienne Boursier}

\section{OpenEdition}

Journals

Édition électronique

URL : http://journals.openedition.org/insitu/18892

DOI : 10.4000/insitu.18892

ISSN : $1630-7305$

Éditeur

Ministère de la culture

Référence électronique

Fabienne Boursier, «Les jardins familiaux des Petits-Bois à Versailles : un patrimoine vivant à

préserver », In Situ [En ligne], 37 | 2018, mis en ligne le 12 décembre 2018, consulté le 19 avril 2019

URL : http://journals.openedition.org/insitu/18892 ; DOI : 10.4000/insitu.18892

Ce document a été généré automatiquement le 19 avril 2019

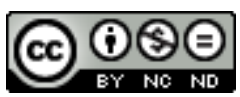

In Situ Revues des patrimoines est mis à disposition selon les termes de la licence Creative Commons Attribution - Pas d'Utilisation Commerciale - Pas de Modification 4.0 International. 


\section{Les jardins familiaux des Petits-Bois à Versailles : un patrimoine vivant à préserver}

The 'Petits-Bois' family gardens at Versailles, a living heritage to be preserved

Fabienne Boursier

Les jardins familiaux des Petits-Bois sont situés au nord-est de la ville de Versailles (Yvelines), dans l'actuel quartier Jussieu-Petits-Bois-Picardie (fig. 1). La présente publication vise à mettre en lumière leurs atouts historiques et contemporains et les difficultés auxquelles ils sont confrontés. Eu égard à leur histoire séculaire, leur inscription dans l'histoire horticole et urbaine est abordée et les raisons de leur récente labellisation « jardin remarquable » sont rappelées. Enfin, l'opportunité est offerte, grâce à une enquête ethnographique menée en $2015^{1}$, d'exposer les caractéristiques du site et de la population des jardiniers, ainsi que leurs pratiques jardinières. 
Figure 1

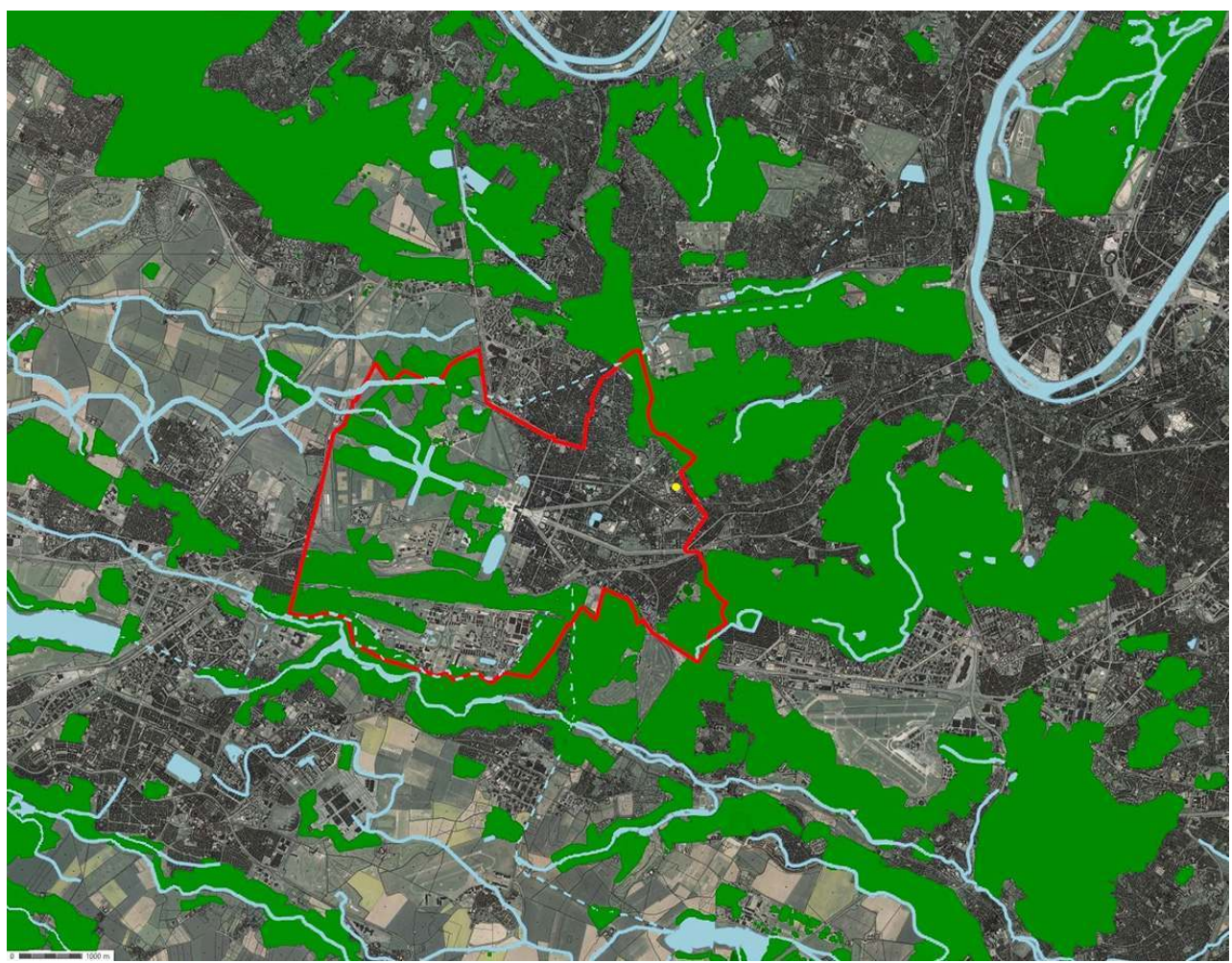

Photographie aérienne, Versailles et ses environs. Les jardins familiaux des Petits-Bois sont figurés par un point jaune.

(C) IGN, 2011 (retouchée).

\section{Une trace vivante de l'histoire horticole et urbaine locale et nationale}

2 Le village de Montreuil, avant de devenir le quartier Jussieu-Petits-Bois-Picardie, rattaché à la ville de Versailles, a été le centre d'une importante production maraîchère et horticole, depuis le XVIII ${ }^{\mathrm{e}}$ siècle $^{2}$ et jusque dans les années 1950. M. Poirier, des établissements horticoles Poirier, domiciliés rue de la Bonne Aventure, dans ce même quartier, fut d'ailleurs le président d'honneur du premier bureau de L'Euvre des jardins ouvriers de Versailles ${ }^{3}$, à l'origine de la création des jardins ouvriers ${ }^{4}$ des Petits-Bois en 19065 . Dans les années 1930, le maraîcher Royer, les pépinières Moser ou encore l'horticulteur Truffaut établissent leurs sièges et leurs cultures dans ce secteur. Parallèlement, l'urbanisation progressive transforme le village en quartier urbain. Les photographies ci-dessous témoignent, d'une part de la physionomie des jardins ouvriers dans les années 1920 (fig. 2), et d'autre part, des derniers champs horticoles du quartier dans les années 1950, alors qu'apparaissent les premières barres HLM (habitations à loyer modéré) (fig. 3). 
Figure 2

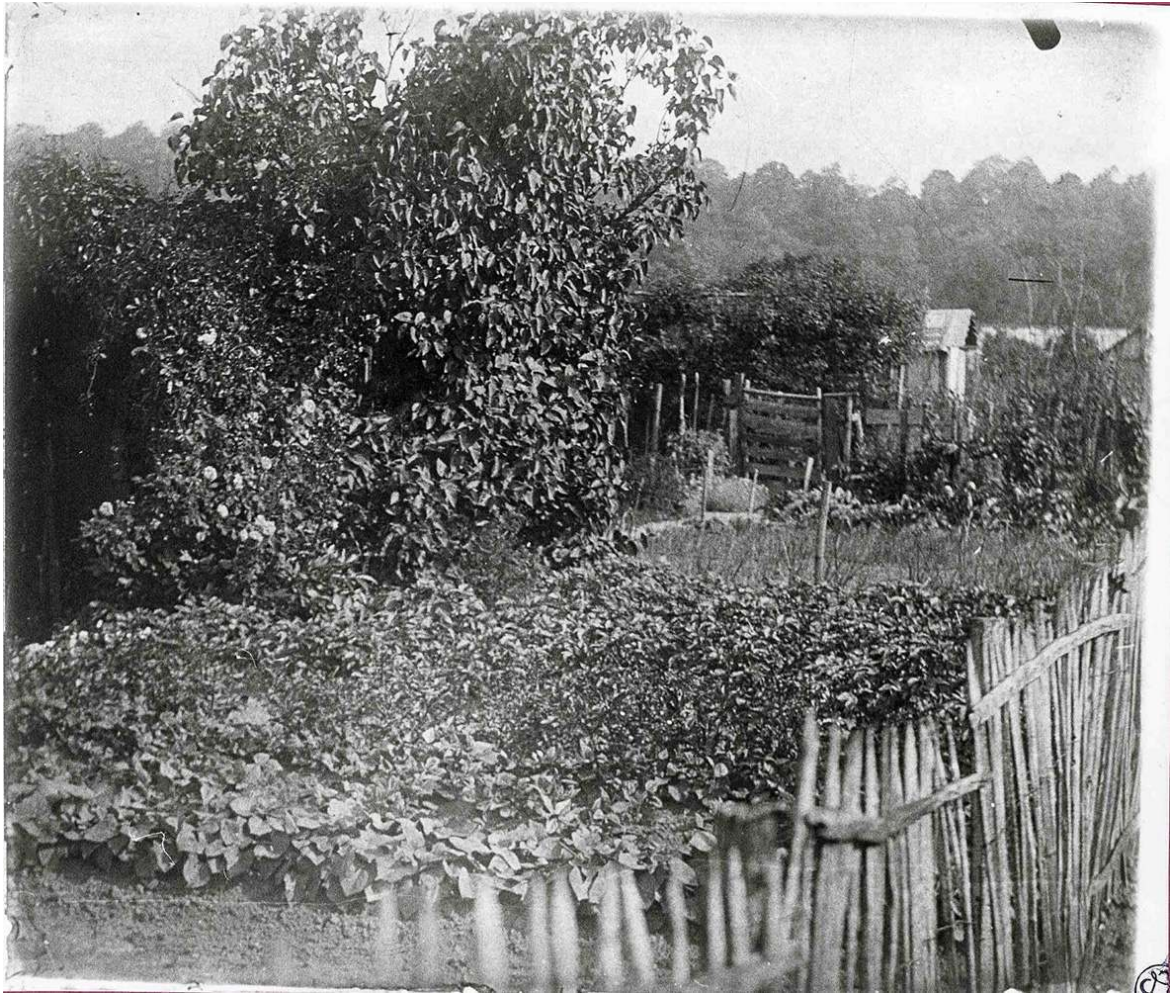

Un jardin parmi les jardins ouvriers des Petits-Bois, Versailles, [1923].

(c) Archives communales (AC) de Versailles, 5 Fi 0402_01.

Figure 3

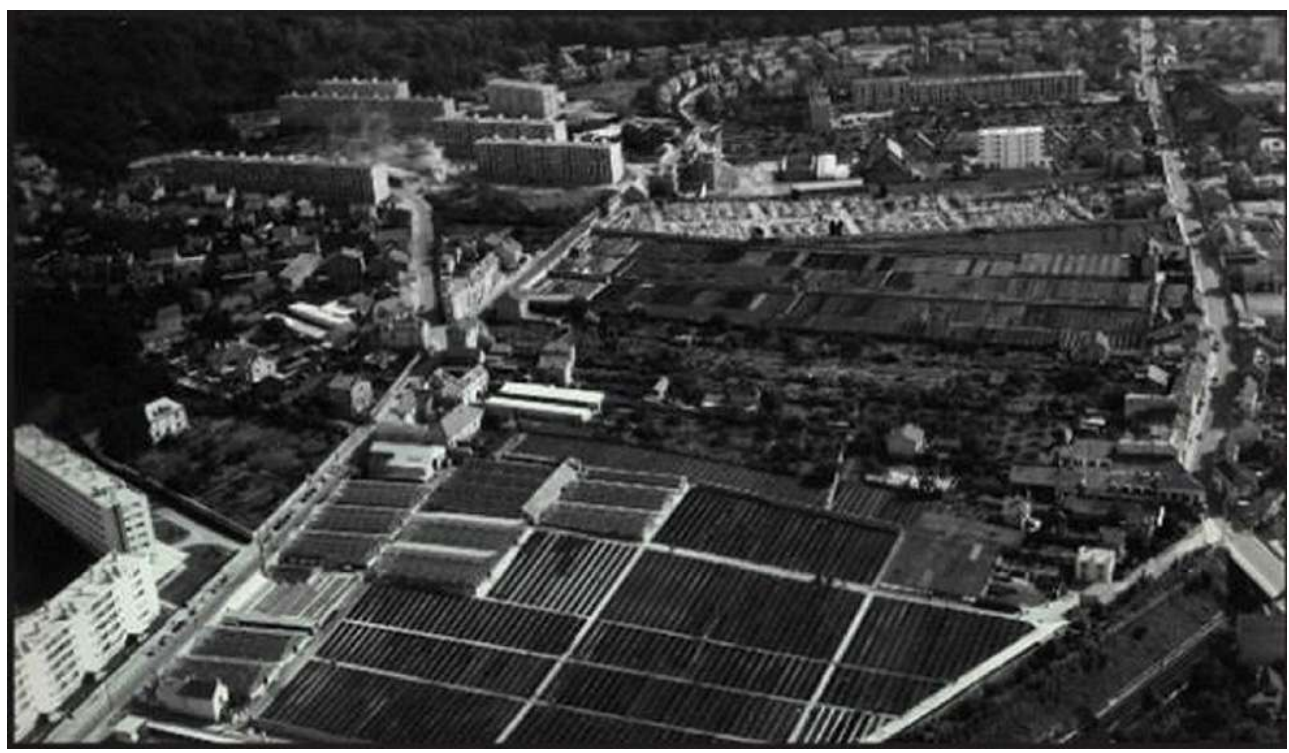

Photographie aérienne prise vers 1954 de l'actuel quartier Jussieu-Petits-Bois-Picardie, à Versailles. On y voit des champs horticoles, les premières barres HLM, et la cité-jardin à l'arrière des jardins familiaux des Petits-Bois.

(c) AC Versailles, 201 Z 5/2. 
Certaines réalisations architecturales et urbanistiques emblématiques subsistent de nos jours autour des jardins familiaux des Petits-Bois. Dans les années 1920, des habitations bon marché (HBM), aujourd'hui détruites, sont érigées sur une partie du terrain des jardins ouvriers. La cité-jardin des Petits-Bois voit le jour au cours des années 1920-1930 (fig. 4). L'église Sainte-Bernadette est bâtie dans les années 1940 (fig. 5), comme le groupe scolaire Albert Thierry. Ces trois dernières réalisations, visibles sur une photographie aérienne de 1954 (voir fig. 8), n'empiètent pas sur le terrain des jardins familiaux, contrairement aux HBM.

\section{Figure 4}

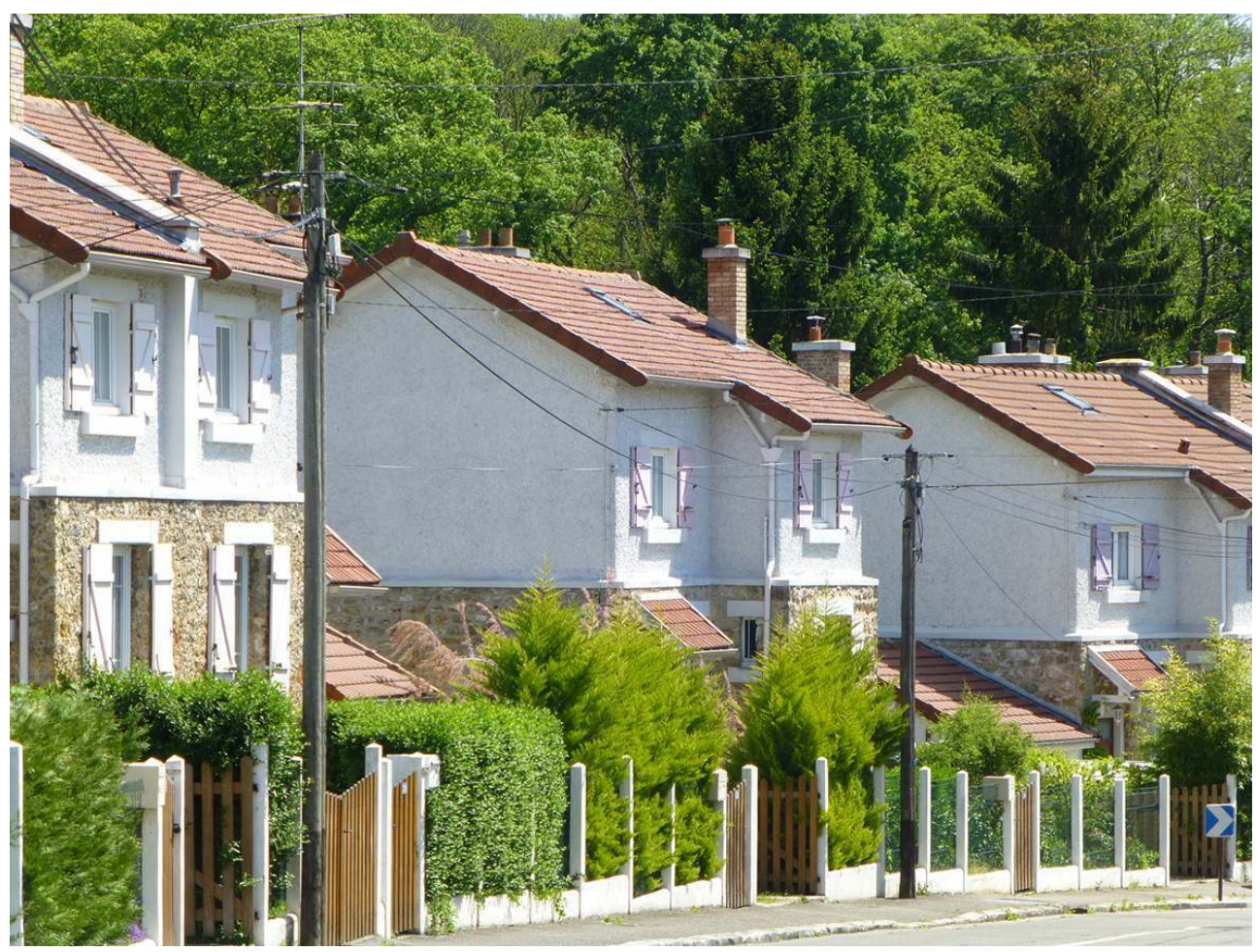

Cité-jardin des Petits-Bois, Versailles, mai 2015.

(c) Fabienne Boursier. 
Figure 5

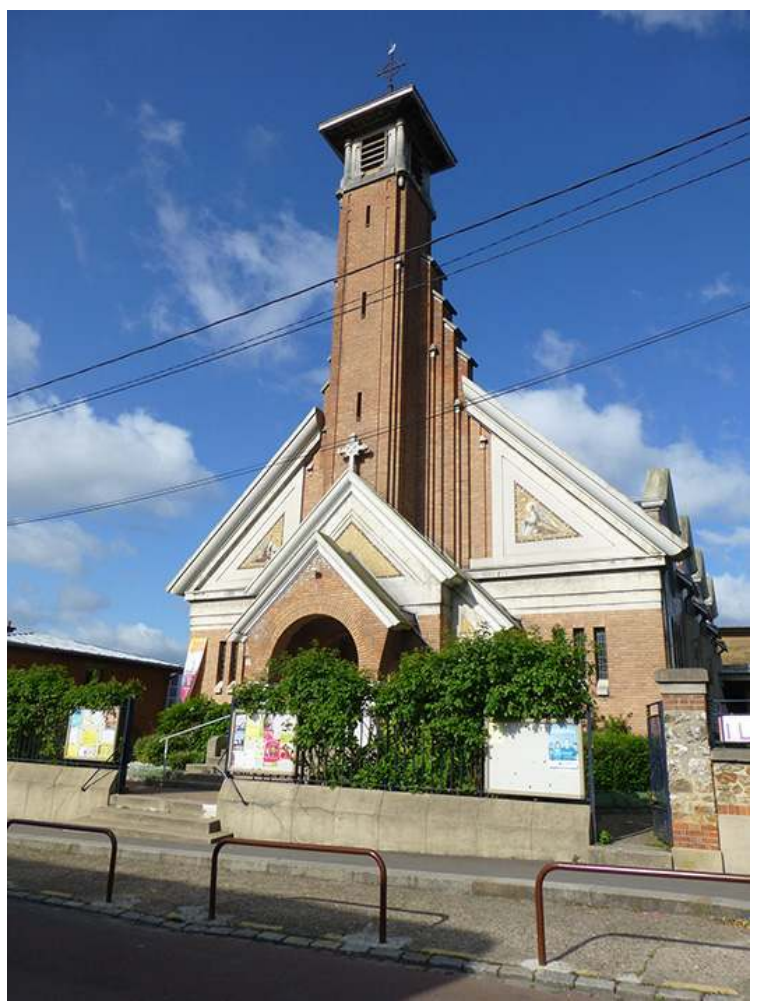

Église Sainte-Bernadette, Versailles, mai 2015.

(c) Fabienne Boursier.

Dans les années 1950-1960, la construction de la cité HLM des Petits-Bois, constituée de 5 immeubles encadrant les jardins, dont certains grignotent le terrain, transforme profondément la physionomie architecturale du quartier. L'environnement devient cloisonné et vertical, du fait de l'apparition et de la multiplication des barres $\operatorname{HLM}^{6}$ (fig. 6, fig. 7). 
Figure 6

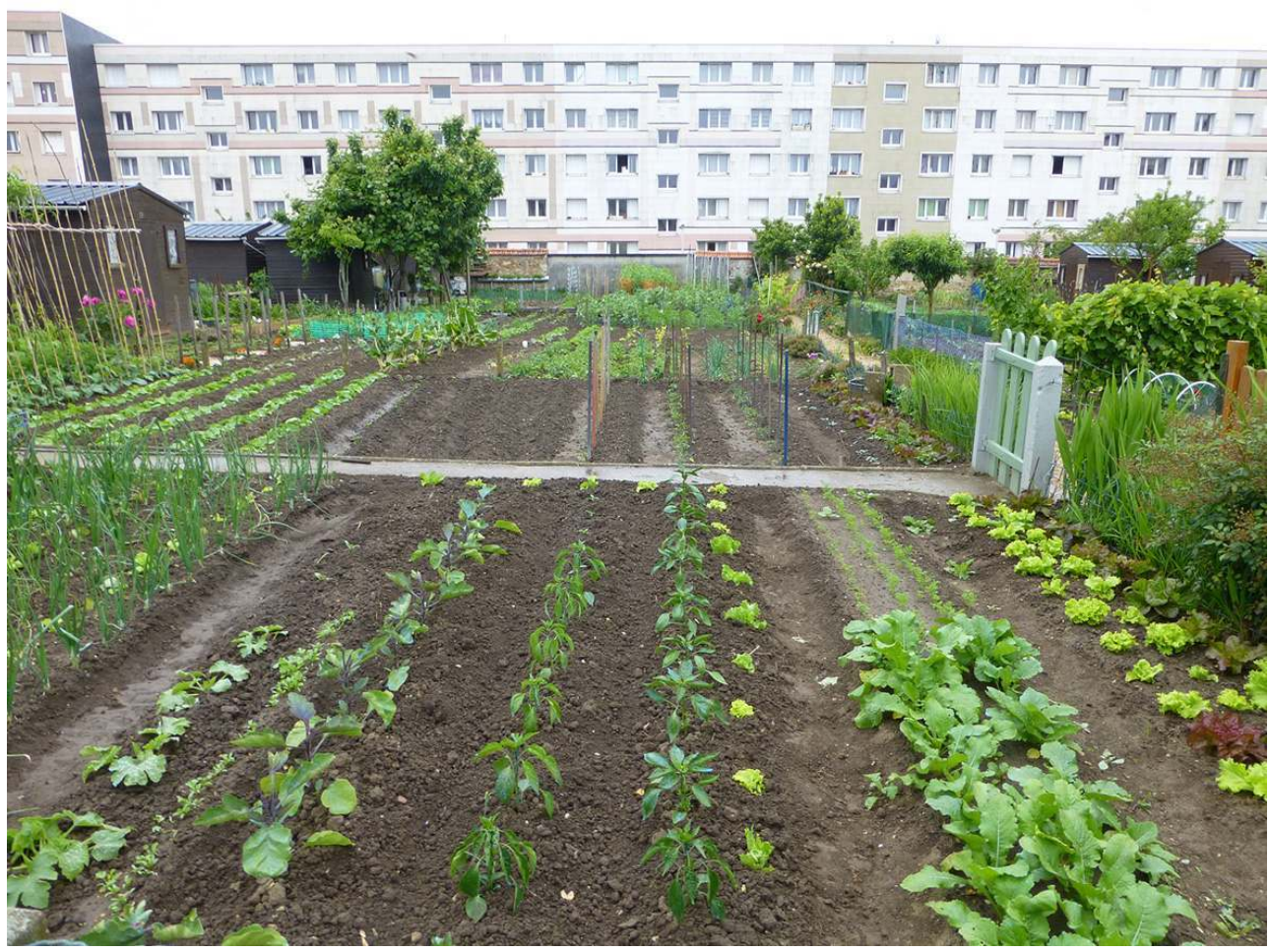

Les jardins familiaux devant un immeuble de la cité HLM des Petits-Bois, Versailles, mai 2015.

(c) Fabienne Boursier. 
Figure 7

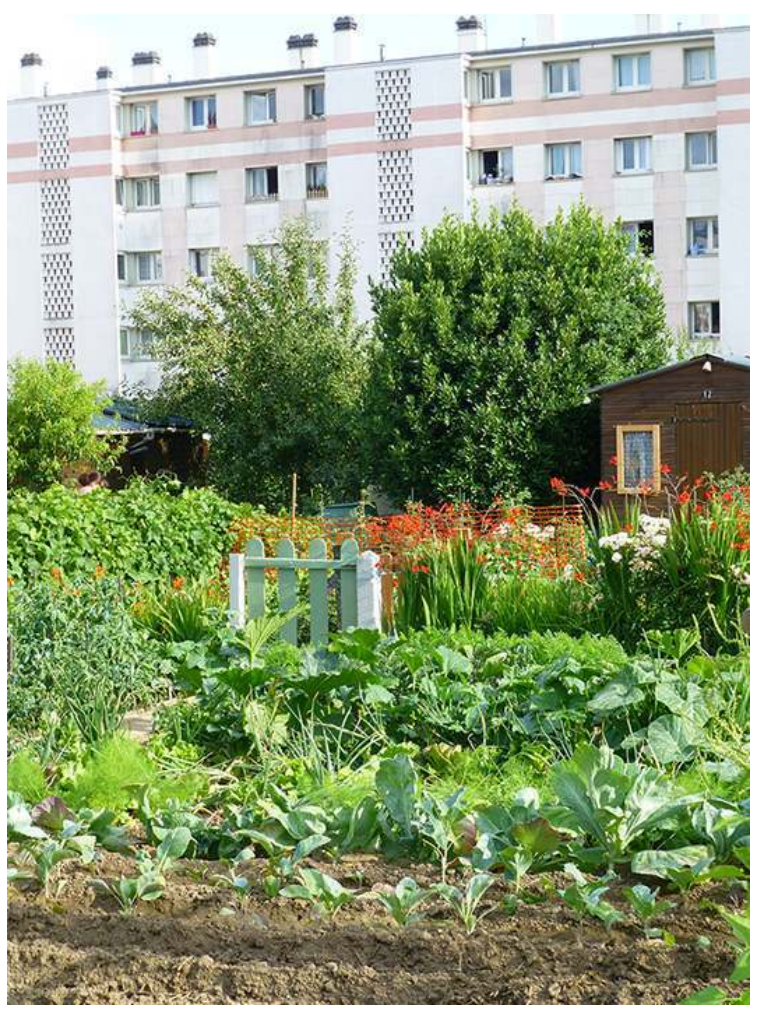

Les jardins familiaux devant un immeuble de la cité HLM des Petits-Bois, Versailles, juillet 2015.

(c) Fabienne Boursier.

Les HBM sont détruites en 1988 et l'immeuble abritant le siège de Versailles Habitat est construit en leur lieu et place. Le gymnase attenant au groupe scolaire de La Source date des années 2010. En 2014, enfin, la maison du gardien, pourtant propriété de l'association gestionnaire au même titre que l'ensemble du terrain des jardins familiaux, est détruite pour permettre de bâtir un immeuble dans lequel, en contrepartie, l'association bénéficie d'un appartement. Plusieurs constructions ont donc progressivement rogné le terrain des jardins familiaux et réduit considérablement leur superficie totale, passée de 1,89 hectare en 1914, lors de la vente du terrain à la Société des jardins ouvriers de Versailles et des communes environnantes ${ }^{7}$, à 1 hectare aujourd'hui (fig. 8). 


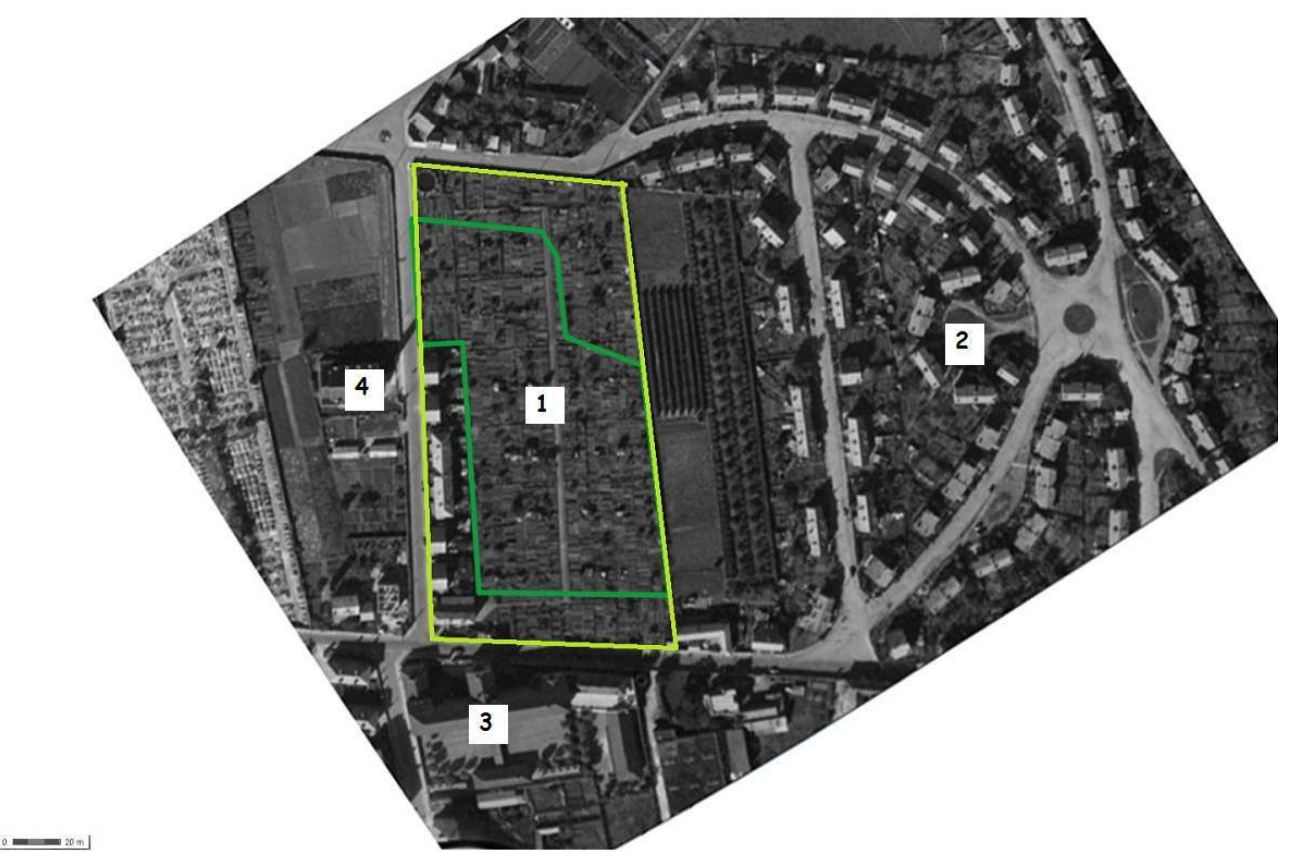

Photographie aérienne, Versailles. Jardins familiaux (1), cité-jardin des Petits-Bois (2), groupe scolaire Albert Thierry (3) et église Sainte-Bernadette (4). Le périmètre du site originel des jardins (1906) est en vert clair, celui du site actuel (2015) est en vert foncé.

\section{De la prise de conscience des jardiniers à la labellisation}

La planification d'un dernier projet immobilier, à l'emplacement de la maison du gardien, a poussé les jardiniers administrateurs à s'interroger sur le statut de l'association, sur l'histoire des jardins et sur leur avenir. Ils ont alors sollicité Pierre Desnos, localement connu comme historien et écrivain. La réduction progressive du terrain des jardins familiaux tout au long de leur histoire, la densité du bâti du quartier (fig. 9) - bien visible depuis les jardins, puisqu'ils sont eux-mêmes entourés de plusieurs immeubles HLM ainsi que la pression foncière incessante les ont sans doute encouragés. Pierre Desnos a répondu à leur appel et décidé, avec l'aide de quelques-uns, d'exploiter le fonds d'archives conservé depuis l'origine par l'association. Il en a tiré un ouvrage sur l'histoire des jardins ${ }^{8}$. Il a ensuite encouragé les membres de l'association à faire don de ce fonds aux archives communales de Versailles. Corinne Hubert, responsable des archives communales, après avoir réalisé l'inventaire $\mathrm{du}$ fonds et trouvant l'ensemble particulièrement intéressant, a pris contact avec Claire Vignes-Dumas, correspondante jardins à la direction régionale des Affaires culturelles d'Île-de-France, et lui a envoyé l'inventaire ainsi que le livre de Pierre Desnos. Claire Vignes-Dumas, après avoir longuement visité les jardins, a présenté le dossier au Conseil national des parcs et jardins ${ }^{9}$ qui a décidé de l'attribution du label «jardin remarquable ». La labellisation a eu lieu en décembre 2014. 


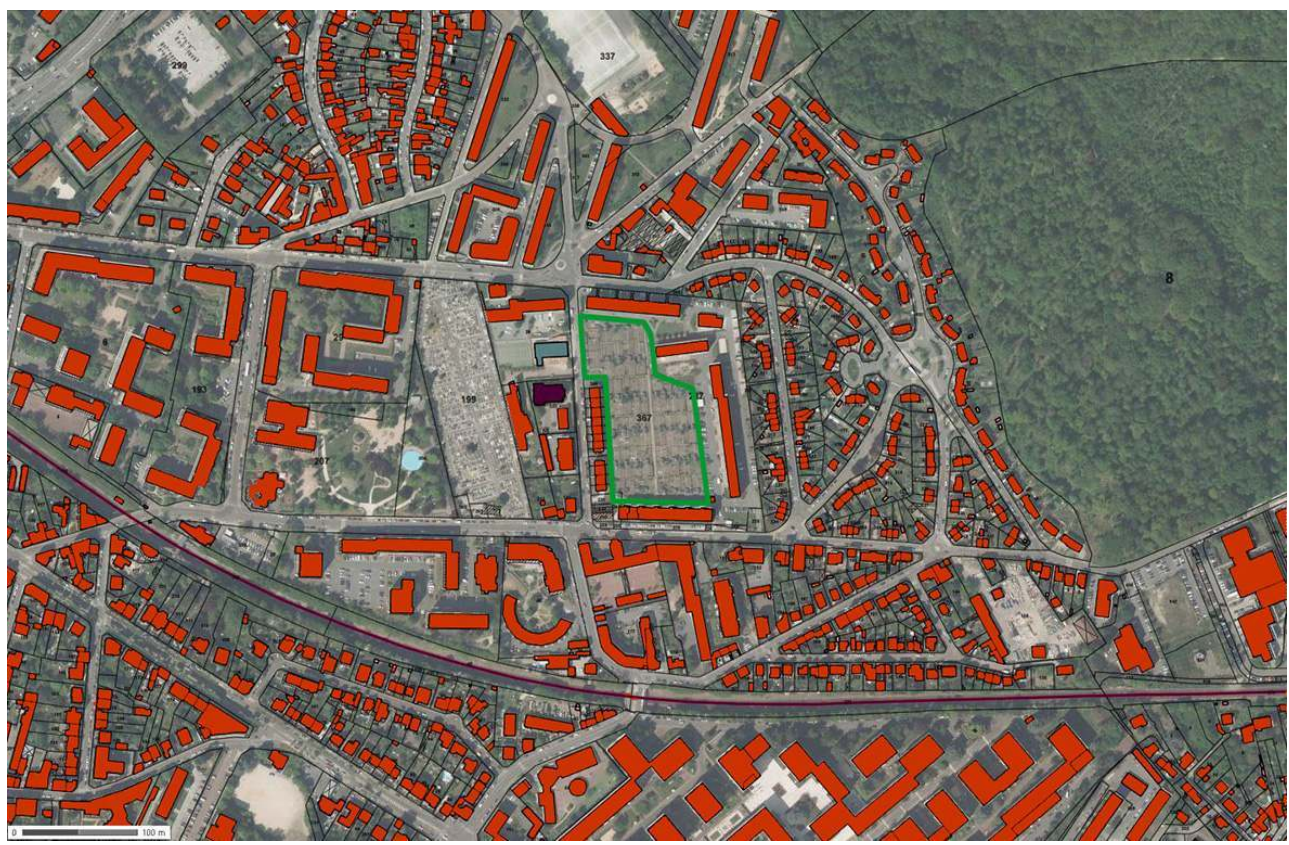

Photographie aérienne, Versailles, quartiers Jussieu-Petits-Bois-Picardie et Montreuil. Le périmètre des jardins familiaux est en vert.

(c) IGN : 2011 (retouchée)

7 Les raisons qui ont conduit à cette labellisation sont de plusieurs ordres. Leur intérêt historique, dont témoigne le fonds d'archives conservé depuis l'origine par l'association, en est la raison principale. Leur caractère vivrier en milieu urbain a également été mis en avant, de même que leurs vertus sociales et environnementales ainsi que la grande variété des végétaux cultivés ${ }^{10}$. Enfin, la volonté de protéger ce patrimoine populaire, et l'espoir que l'on ne puisse plus, à l'avenir, considérer les jardins familiaux comme une simple réserve foncière, sont deux autres raisons non moins importantes. L'identification des jardins familiaux des Petits-Bois comme terrains cultivés, donc protégés, sur le plan de la trame verte du PLU ${ }^{11}$ de Versailles en 2006, n'avait pas empêché la construction d'un immeuble sur le site de la maison du gardien. La pression foncière risquait de s'intensifier encore du fait de l'adoption de la loi dite Alur ${ }^{12}$ visant à transférer les compétences en matière d'urbanisme aux intercommunalités, à encourager la densification en zone urbaine et à mobiliser les gisements fonciers ${ }^{13}$. La menace pesant sur ces jardins familiaux a contribué à la décision de leur labellisation. Celle-ci devrait à son tour induire des actions de coopération et renforcer des "phénomènes d'appropriation ${ }^{14}$. D'ailleurs, malgré les contraintes découlant de cette distinction, comme la visibilité accrue des jardins et l'obligation de leur ouverture au public, la plupart des jardiniers la considèrent avec fierté :

Arrouch : « C'est bien pour le quartier, on devrait faire ça partout. »

Nicolas: «Au niveau historique, c'est une association qui ne date pas d'hier, c'est une volonté aussi. Je pense que les jardins familiaux ont risqué à plusieurs reprises de ne plus exister. Donc s'ils existent encore aujourd'hui, c'est parce qu'il y a des gens qui se sont battus, qui ont été volontaires, qui y ont cru, et franchement, je trouve ça très beau. "

Pascal : «Ce serait dommage que ça disparaisse pour les générations futures. C'est bien qu'on puisse continuer, c'est une tradition. Et puis, le jardin, c'est quand même 
typiquement français. »

Teresa : «Parce qu'il est beau et d'utilité publique, voilà ! Il y a plein de choses !»

Le site s'étend donc désormais sur 1 hectare, il est légèrement en pente et comporte 80 parcelles de jardinage. Chaque parcelle a une superficie allant de 100 à $150 \mathrm{~m}^{2}$ et est dotée d'un abri de jardin. La plupart des cabanes de jardin ont été uniformisées dans les années 1990 pour remplacer les abris de fortune. Les plus récentes datent de 2012. Pendant l'enquête, certains jardiniers ont mentionné la qualité non homogène de la terre dans l'enceinte des jardins. Certaines zones seraient en effet moins fertiles que d'autres. D'après le jardinier Arrouch, les courgettes, les potirons, les betteraves poussent n'importe où sur sa parcelle. En revanche, les tomates et les pommes de terre «crèvent au bout d'un mois » à certains endroits. Il ne l'explique pas, mais adapte ses rotations de culture en fonction de ces observations. Il remarque également qu'avec $150 \mathrm{~m}^{2}$, on ne peut pas faire reposer la terre. Par ailleurs, plusieurs jardiniers se sont plaints de récoltes moins bonnes depuis plusieurs années. Certains attribuent cela au climat, d'autres à la terre, qui s'épuiserait à force d'être cultivée. Des jardiniers ont témoigné d'apports de terre à différentes époques. Il semblerait d'ailleurs qu'à la suite de la destruction des maisons HBM à la fin des années 1980, des remblais aient été enfouis sous les jardins. Daniel retrouve ainsi des gravats lorsqu'il creuse un peu plus profondément. De façon générale, il serait intéressant de situer précisément les zones moins fertiles identifiées par les jardiniers et, avec une aide institutionnelle, de procéder à des sondages. Quant aux pratiques en matière de rotation des cultures et d'amendement du sol, il est souhaitable de les encourager. Nous y reviendrons dans la partie consacrée aux pratiques jardinières. Les accès à l'eau, 29 points répartis sur le site de façon équilibrée, sont aujourd'hui branchés sur le réseau d'adduction d'eau de la ville (fig. 10). Mais l'alimentation pourrait se faire directement à partir de la nappe phréatique. En effet, il est précisé sur l'acte de vente du terrain d'avril 1914 qu'il comprend « entre autres 7 puits et un bassin servant de réservoir d'eau ${ }^{16}$. Cette éventualité nécessiterait néanmoins des travaux de gros œuvre et, par conséquent, la sollicitation d'institutions locales ou nationales afin d'obtenir des aides financières. En effet, en vertu des statuts des jardins familiaux édictés dans le Code rural $^{17}$, les associations gestionnaires peuvent prétendre à des subventions destinées à leur aménagement, de la part de l'État ou de collectivités territoriales locales. 


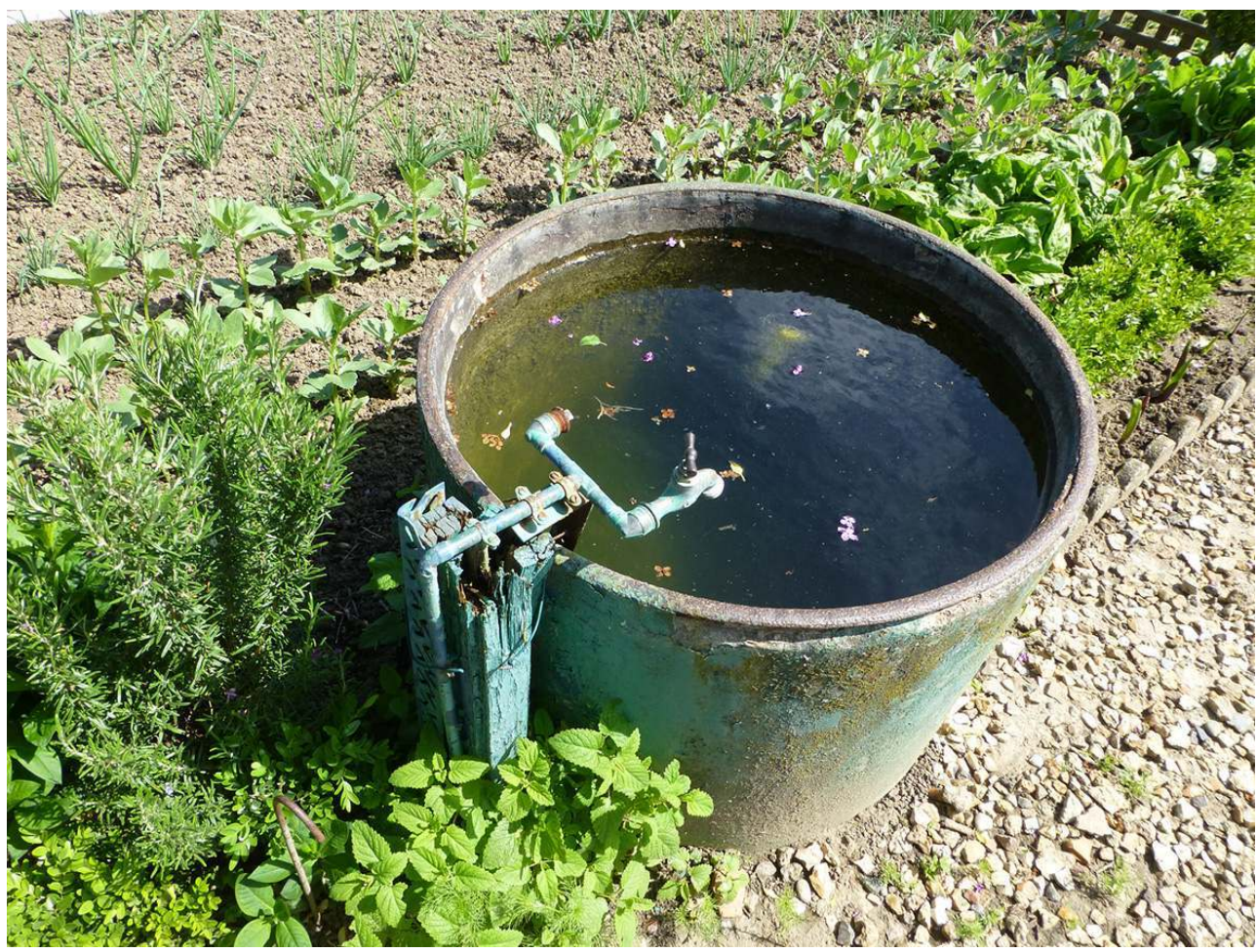

Point d'eau dans l'enceinte des jardins familiaux des Petits-Bois, Versailles, mai 2015

\section{La population des jardiniers : une photographie sociale}

Selon les informations fournies par Marcel Saintonge, président de l'association gestionnaire des jardins familiaux des Petits-Bois, sur 79 jardiniers ${ }^{18}$, les plus nombreux sont les 22 cinquantenaires, suivent les 17 soixantenaires, les 15 quarantenaires, les 10 septuagénaires, les 5 octogénaires et les 4 trentenaires (l'âge de 6 jardiniers n'est pas connu). Le plus jeune des jardiniers a 30 ans et la plus âgée 88 . Ce site est donc intergénérationnel. 49 sont des hommes et 27 des femmes, 3 des jardins cultivés l'étant par des couples (par exemple : mari et femme ou mère et fille). La proportion des femmes a augmenté depuis $2011^{19}$. Et, comparativement aux enquêtes menées dans les années 1970 par Françoise Dubost à Créteil, cela représente une augmentation considérable du nombre de femmes cultivant les jardins. Pourtant, sur le site de la Ménagerie, géré par la même association et situé à la périphérie de la ville, les chiffres donnent 32 hommes pour 2 femmes. Aux Petits-Bois, la doyenne des jardiniers cultive son jardin depuis 55 ans et le benjamin depuis 1 an. La grande majorité des jardiniers, soit 69 personnes, habitent Versailles mais pas forcément le quartier. 5 personnes habitent Viroflay, 3 Vélizy-Villacoublay, 1 personne Guyancourt et 1 autre Magny-les-Hameaux. Tous résident dans le département des Yvelines. Concernant l'origine géographique des jardiniers, selon les informations fournies par l'association en 2015, plus de la moitié (49) sont originaires de France, 13 du Maghreb, 10 du Portugal, 3 de Pologne, 2 du Laos, et enfin 2 d'îles de l'océan Indien (île de La Réunion et île Maurice). La répartition de 
l'origine géographique des jardiniers a légèrement évolué depuis 2011, la proportion des jardiniers d'origine étrangère ayant légèrement augmenté en quatre ans. Les Italiens présents dans les jardins familiaux de Créteil dans les années 1970 ne le sont plus aux Petits-Bois à Versailles en 2015, mais l'étaient encore de façon minoritaire en 2011. Comparativement à 2011, les Maghrébins sont un peu moins nombreux et les Portugais et Polonais de plus en plus nombreux. Notons la présence de Laotiens, Mauriciens et Réunionnais, et l'absence de jardiniers originaires d'Afrique noire. Il n'est pas possible d'établir la profession d'origine des retraités, qui constituent pourtant le groupe le plus nombreux (31 personnes). Néanmoins, nous pouvons établir le profil professionnel de quelques-uns d'entre eux, sans connaître précisément leur emploi (ouvrier, technicien, employé ou cadre), grâce aux entretiens ou aux informations fournies par le président de l'association. Cinq ont ainsi travaillé dans l'industrie automobile, un à la SNCF, un pour EDF, un à la Ville de Versailles, un dans les travaux publics, un dans le secteur hospitalier, deux sont des artisans (un plombier et un horloger). Parmi les actifs, il y a 27 employés et techniciens ${ }^{20}$ et seulement 7 ouvriers et ouvriers qualifiés, il y a encore 7 cadres, 4 sans emploi, et 1 jardinier exerce un métier artistique (la profession de 2 jardiniers n'est pas connue). Par rapport à 2011, le nombre de retraités a donc légèrement diminué et le nombre de cadres augmenté. L'absence d'ouvriers en 2011 tient peut-être au fait qu'ils n'ont pas été distingués des employés. De façon générale, et par rapport aux années 1970, les ouvriers sont beaucoup moins représentés, contrairement aux employés qui ont considérablement augmenté. Globalement, ces données reflètent le vieillissement de la population, mais aussi l'évolution du tissu économique français et plus particulièrement, celui de l'île-de-France, où le secteur secondaire est sous-représenté par rapport au secteur tertiaire. L'enquête menée en 2015 auprès de 16 jardiniers des Petits-Bois ( 7 femmes et 9 hommes), soit un cinquième de l'ensemble, nous a permis d'obtenir des informations plus précises. Nous avons également interviewé 2 habitants de la cité HLM des Petits-Bois ( 1 jeune homme et 1 femme à la retraite). Selon les récits des personnes interrogées, la proportion des jardiniers d'origine rurale demeure importante. Par ailleurs, parmi les 18 personnes interrogées, 12 habitent le quartier, et selon nos déductions, une majorité vivrait en appartement (dont 4 dans l'un des immeubles de la cité HLM des Petits-Bois encadrant les jardins). Quelques-uns vivent en pavillon, l'un d'entre eux ayant même un terrain. Le profil des personnes interrogées dans le cadre de l'enquête correspond globalement au profil moyen de l'ensemble des jardiniers. Lorsqu'il varie sensiblement (par exemple pour les tranches d'âge ou l'origine géographique), cela est dû à l'impossibilité d'interroger certaines personnes. L'une des raisons alors invoquée par les jardiniers était le manque de temps (souvent le cas pour les plus jeunes), mais la méfiance ou le manque de confiance en soi peuvent également avoir été à l'origine du refus de participer à l'enquête. L'âge des personnes interrogées a été systématiquement noté au début de chaque entretien. La profession n'est en revanche pas mentionnée. Mais elle a pu être évoquée pendant les entretiens. Elle est en outre mentionnée pour les actifs et parfois pour les retraités dans les documents fournis par le président de l'association. Quand les informations fournies par l'association étaient en contradiction avec celles recueillies lors des entretiens, nous avons privilégié le témoignage oral. Ainsi, Andrée, 88 ans, est retraitée, Marcel, 60 ans, est technicien de l'industrie automobile retraité, Daniel, 70 ans, est horloger retraité, Arrouch, 68 ans, est plombier retraité, Pascal, 50 ans, est jardinier professionnel (entretien d'espaces verts), Yann, 52 ans, travaille dans une chaufferie, Dominique, 56 ans, est technicien de l'industrie automobile, Nicolas, 30 ans, est cadre, Stéphanie, 40 ans, est cadre, Jean, 57 ans, est menuisier, Fernand, 51 ans, est 
employé dans le commerce, Jeanine, 81 ans, est retraitée, Sophie, 45 ans, est employée ou cadre, Annick, 71 ans, est retraitée, Teresa, 52 ans, est aide-soignante ou infirmière, Yvonne, 73 ans, est employée d'hôpital retraitée. En ce qui concerne les 2 habitants des HLM interrogés, il s'agit de Stephen, 19 ans, en apprentissage dans la restauration, dont la grand-mère habite un pavillon de la cité-jardin des Petits-Bois, où elle cultive un jardin, et de Denise, 76 ans, femme de ménage retraitée qui profite parfois des produits du jardin grâce aux dons d'Andrée.

\section{Les pratiques jardinières : un riche savoir-faire}

\section{L'apprentissage et la transmission du savoir-faire jardinier}

11 L'apprentissage du jardinage s'est fait de différentes façons selon les personnes. Certaines ont appris durant l'enfance auprès de leurs parents cultivateurs, comme Yvonne, Annick ou Teresa, mais cela représentait plus une contrainte qu'un véritable plaisir. Les travaux agricoles étaient durs, mais la transmission du travail de la terre a bien eu lieu. Et c'est seulement à l'âge adulte qu'elles ont vraiment pris goût au jardinage. D'autres ont assisté leurs parents ou leurs grands-parents au jardin, tels Andrée, Marcel, Jeanine, Sophie, Stéphanie ou Arrouch. Andrée, enfant, avait son propre carré de terrain à cultiver. Stéphanie a décidé de jardiner après avoir entendu un témoignage de Pierre Rabhi, spécialiste de l'agro-écologie, dans le documentaire réalisé par Coline Serreau Solutions locales pour un désordre global (2010). Dans sa jeunesse, Nicolas a observé sa grand-mère et attendu l'âge adulte pour se lancer à son tour. C'est au contact de personnes de leur entourage (ami, voisin, beaux-parents, frère...) que d'autres ont découvert plus tard le jardinage, comme Dominique, Daniel, Fernand, Yann, Pascal ou Jean. Pascal entretient, en tant que jardinier, le parc d'un immeuble privé depuis vingt ans, mais il n'avait jamais pratiqué le jardin potager avant de trouver une parcelle aux Petits-Bois, en 2009. Jean a appris à jardiner en observant ses semblables et en expérimentant. Les jardiniers échangent entre eux et comparent leurs pratiques respectives. L'entraide, l'échange de pratiques et la transmission existent dans l'enceinte des jardins, entre les plus jeunes et les plus âgés, entre les novices et les plus expérimentés, en fonction des affinités et des domaines de prédilection de chacun. Des besoins en formation ont cependant été formulés, souvent par les plus jeunes. La plupart des jardiniers jardinent seuls, mais apprécient la convivialité permise dans le cadre des jardins familiaux. Certains expérimentent plus que d'autres, comme Teresa ou Jean. Cela n'est apparemment pas lié à l'âge, ni à l'expérience, mais à une appétence particulière. Au fil des entretiens, nous avons pu relever des références d'ouvrages utilisés comme aide au jardinage, tels que Le Parfait jardinier, Le poireau préfere les fraises, Les meilleures associations de plantes, Les tomates aiment les carottes: les secrets $d u$ bon voisinage des plantes dans votre jardin. Le magazine Rustica a été cité à plusieurs reprises, ainsi que l'émission de télévision Silence ça pousse! Le site internet Jardiner avec la lune semble être consulté plus que d'autres, même si la recherche d'informations sur la toile est souvent pratiquée de façon aléatoire.

\section{Les produits utilisés (graines, engrais et répulsifs « naturels »)}

12 L'achat des graines se fait chez différents fournisseurs : la jardinerie de Bois-d'Arcy, la ferme de Gally, les serres de Noisy, les jardineries Truffaut et Jardiland, des jardineries en 
province (régions d'origine) et des grandes surfaces telles que Leclerc ou les Magasins U. Certains jardiniers achètent leurs graines par correspondance, via Kokopelli, Willemse, Fabre ou Jardin express. Auparavant, tous les jardiniers achetaient leurs graines dans une coopérative du département de l'Aisne, mais ce n'est plus le cas aujourd'hui. Certains récupèrent des graines, d'une année sur l'autre, comme Jean, Fernand, Annick, Yann, Arrouch, Dominique et Yvonne. Arrouch, Jean, Fernand ou Marcel en ramènent de leur pays d'origine ou de celui de leur conjointe (Portugal, Algérie, Asie du Sud-Est...). Annick, Yvonne, Fernand, Teresa, Jean et Pascal font leurs plants (fig. 11) et Teresa fait même des boutures, ce qui est plus rare. Pascal précise que pour récupérer les graines et faire ses propres plants, il faut du temps, du savoir-faire et de la patience, mais que cela est gage d'économies substantielles. Le budget annuel des jardiniers n'est d'ailleurs pas aisé à établir. Hormis les 70 euros de cotisation annuelle, la plupart des jardiniers n'établit pas de comptabilité précise.

Figure 11

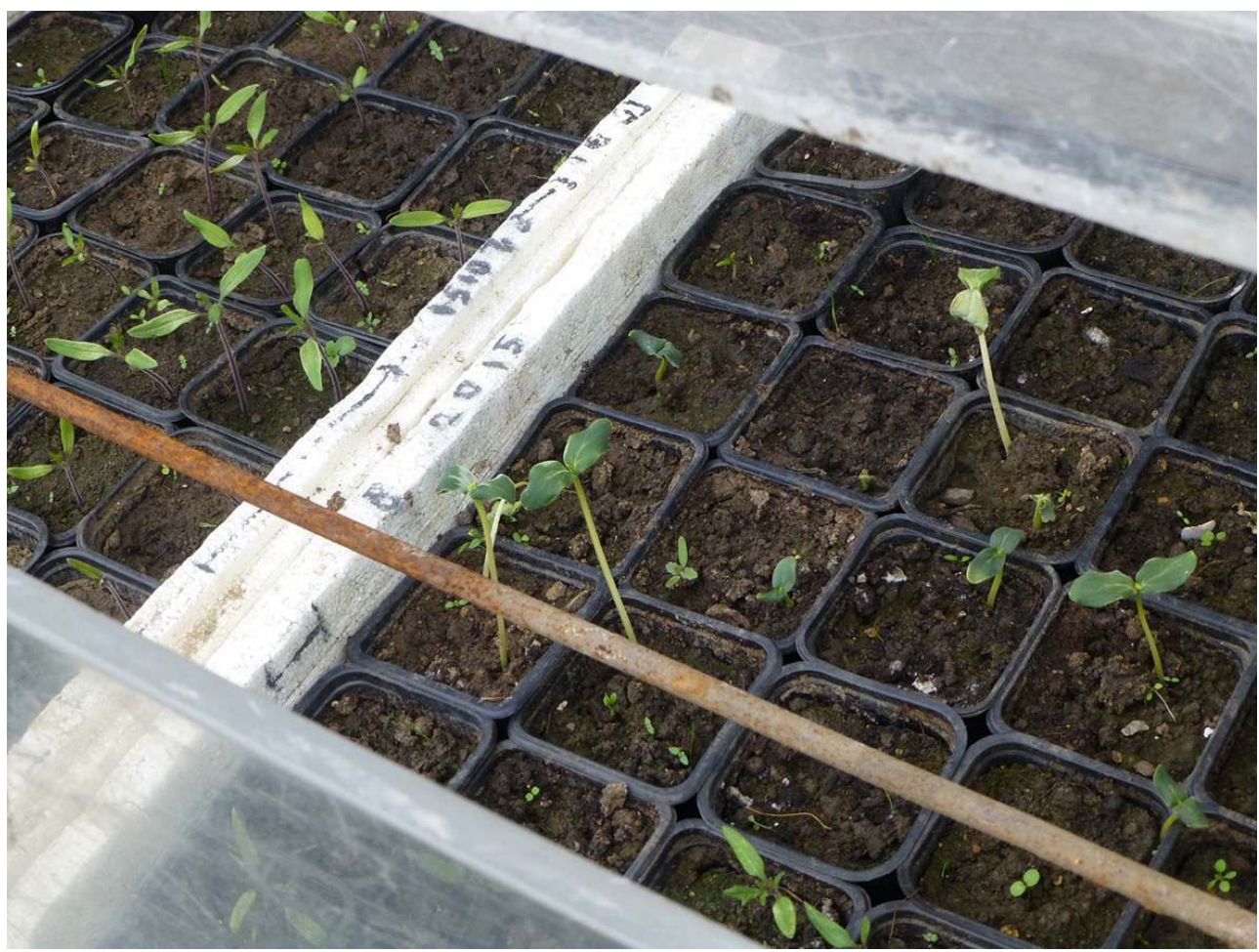

Plants préparés par Jean, jardins familiaux des Petits-Bois, Versailles, avril 2015.

(c) Fabienne Boursier.

13 En matière de pratiques respectueuses de l'environnement, les jardiniers peuvent s'appuyer sur un fascicule édité par la Ville de Versailles ${ }^{21}$. Il est à disposition gratuitement au service des Espaces verts de la ville. Ce service, sous l'impulsion de son actuelle responsable Cathy Biass-Morin, proscrit d'ailleurs l'utilisation des produits phytosanitaires et prône le « zéro phyto » dans les jardins publics et collectifs de la ville. Les jardiniers des Petits-Bois sont donc censés jardiner en respectant ces consignes. Et force est de constater que de nombreux jardiniers usent de pratiques traditionnelles pour cultiver leur parcelle, sans avoir recours aux produits phytosanitaires. Pour protéger ses plantations, Teresa utilise la consoude, qu'elle plante et récupère pour faire du purin, un engrais naturel stimulant la croissance des légumes. Elle utilise également le vinaigre 
blanc pour désherber, éloigner les fourmis et nettoyer ses ustensiles, le savon noir pour ses vertus antibactériennes et insecticides, la camomille qui attire certains insectes et en repousse d'autres, et la valériane qui, selon elle, en infusion aspergée sur les plantes, protège du froid. Andrée plante des œillets d'Inde entre ses tomates pour éviter que les papillons n'y pondent leurs œufs. Elle plante le poireau à côté du fraisier, ainsi que la capucine avec les courgettes, car elle attire le puceron et la punaise de la courge. Annick, depuis vingt ans dans les jardins, reconnaît avoir utilisé, par le passé, certains produits phytosanitaires, mais plus depuis leur interdiction. Les mentalités ont donc évolué. Certains reconnaissent utiliser parfois des granulés anti-limaces. Andrée et Marcel, pour faire fuir ces gastéropodes, insèrent un fil de cuivre dans les salades qu'ils repiquent. Quasiment tous les jardiniers utilisent la bouillie bordelaise, fongicide composé d'eau, de sulfate de cuivre et de chaux, autorisé en agriculture biologique. Pascal utilise de l'or brun, à base d'algues, un engrais naturel à mélanger à petite dose au terreau, «cela fortifie les légumes et les rend résistants ». Avant qu'ils soient officiellement interdits en France, certains jardiniers utilisaient des produits «bio " «neem ${ }^{22} »$. Le purin d'ortie, excellent engrais naturel et antiparasitaire, acheté ou réalisé par soi-même, est également souvent utilisé. Marcel dépose du paillage pour empêcher l'herbe de pousser, Jeanine désherbe à la pelle et Yann utilise le gros sel. Jean a recours à la grelinette pour retourner la terre en surface. Il arrive que les jardiniers expérimentent des modes de culture, à l'instar des colonnes verticales créées spécialement pour les fraisiers par Daniel et Fernand.

\section{L'amendement de la terre et la rotation des cultures}

Des formations sont proposées par la Ville depuis plusieurs années pour favoriser l'utilisation de composteurs, mis gratuitement à disposition de chaque jardinier. Aujourd'hui, la plupart en sont équipés. Mais de nombreux jardiniers utilisaient déjà du compost et amendaient le sol auparavant, chacun à sa manière. Le terreau acheté en jardinerie est utilisé, mais pas uniquement. L'enfouissement de marc de café, mélangé à la terre au pied de certaines plantes, est souvent pratiqué. L'année précédente, Pascal a étalé sur son terrain, en guise de compost, 1,8 tonne (une centaine de sacs) de feuilles et de restes de tonte, « une fois mélangé à la terre, cela agit au bout de quelques années ». Fernand récupère également des tontes de gazon et des feuillages auprès de proches ou de jardiniers de son quartier. Teresa utilise du compost, mais pas systématiquement, « les oignons n'aiment pas, par contre pour les pommes de terre et les tomates, il faut en mettre ». Yann nous rappelle qu'auparavant, quand les feux étaient autorisés sur les parcelles pour le nettoyage, les cendres étaient réparties sur le terrain pour le fertiliser. Arrouch préfère le terreau et le fumier de cheval au compost. Il précise que le fumier ne doit pas être utilisé avant une année après sa production, à cause de sa haute teneur en sel. Nicolas utilise du fumier de cheval et cultive sur des buttes de terre. La plupart des jardiniers interviewés pratiquent la rotation des cultures. Mais, pour promouvoir durablement cette pratique et la généraliser, des formations pourraient avoir lieu entre jardiniers ou avec une aide extérieure.

\section{Les différends entre les jardiniers et les incivilités}

L'utilisation de l'eau est, par ailleurs, source de tensions et de désaccords. Certains jardiniers pratiquent l'arrosage en fin de journée, avec parcimonie, et considèrent que 
d'autres la gaspillent. Il est d'ailleurs précisé dans le règlement intérieur que l'arrosage ne doit se faire qu'à l'arrosoir, et non au jet. Yvonne et Annick utilisent des réservoirs pour récupérer l'eau de pluie. Cette pratique pertinente devrait être encouragée sur l'ensemble des parcelles comme le suggère Jeanine. L'autre différend concerne la multiplication de l'utilisation des serres. De petites serres peuvent être utilisées pour faire des plans, mais il est précisé dans le règlement intérieur que les châssis ou tunnels ne doivent pas dépasser un mètre de hauteur. Pourtant, à la suite de plusieurs mauvaises saisons, quelques jardiniers ont installé, notamment pour la culture des tomates, des serres un peu plus hautes qu'à l'accoutumée, tout en s'engageant à les démonter au terme de la saison (fig. 12). D'autres y sont farouchement opposés, en raison de leur aspect disgracieux et de leur inutilité. Malheureusement, ces désaccords entre jardiniers, le nonrespect du règlement intérieur, tout comme les incivilités répétées ou le désengagement de plus en plus courant des jardiniers vis-à-vis de la gestion associative, qui repose sur un petit nombre de personnes, pourraient représenter une autre menace pour les jardins. Par ailleurs, Dominique déplore des jets de détritus sur sa parcelle depuis l'immeuble qui la surplombe. Néanmoins, malgré des dégradations et vols survenus quelques années auparavant dans l'enceinte des jardins, ils sont généralement appréciés et respectés du voisinage.

Figure 12

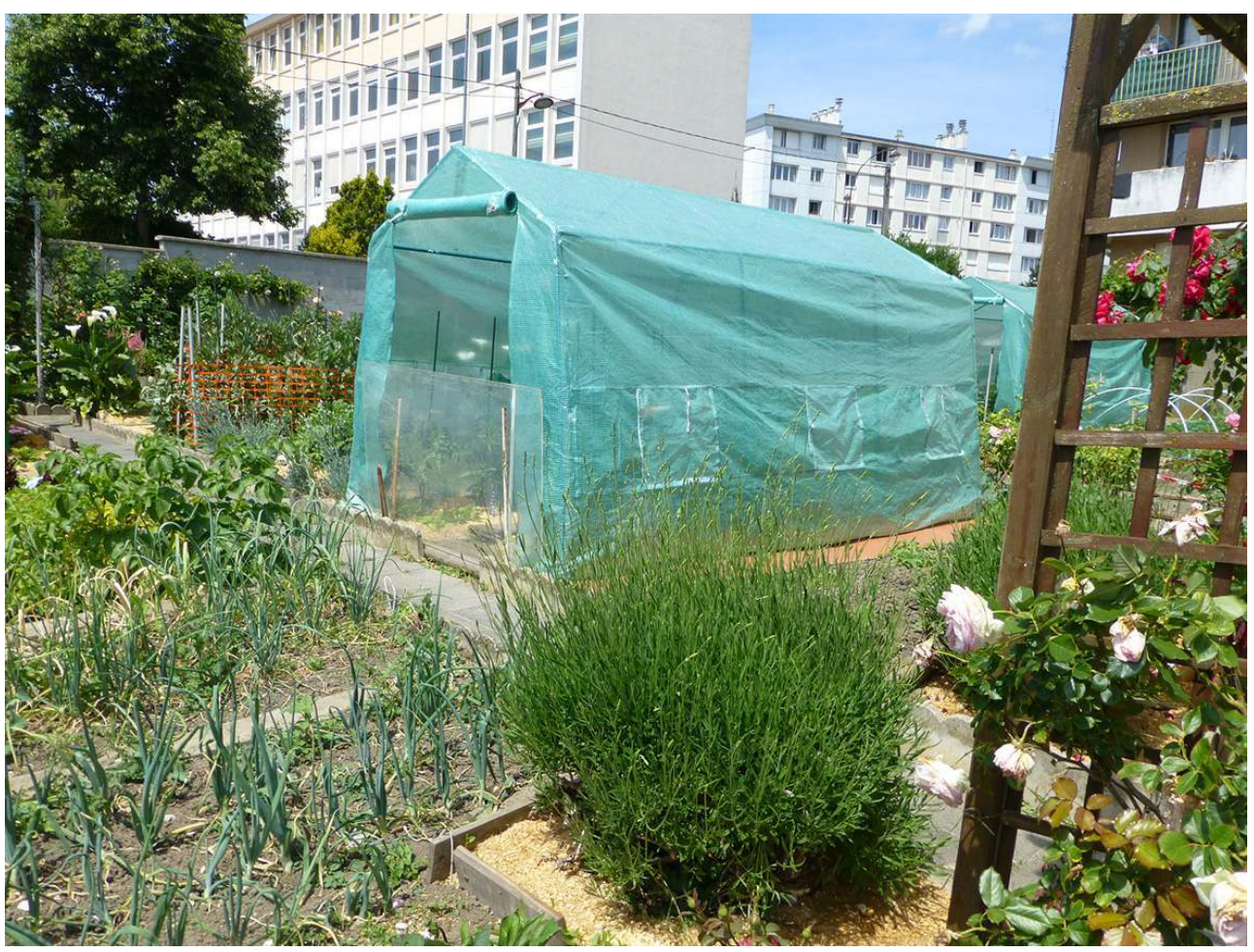

Deux serres provisoires installées pour la culture des tomates dans les jardins familiaux des PetitsBois à Versailles, juin 2015.

(c) Fabienne Boursier. 


\section{La grande variété des plantes cultivées}

16 Parallèlement à l'enquête initiale menée auprès des 18 personnes, une seconde a été menée auprès de 56 jardiniers afin de mieux connaître les espèces et variétés de plantes cultivées dans les jardins. Il s'agissait de cocher et compléter un tableau type ${ }^{23}$. Il est apparu qu'environ 200 espèces et 580 variétés sont cultivées dans les jardins familiaux des Petits-Bois, soit 49 espèces et 324 variétés de légumes, 38 espèces et 61 variétés de condiments (herbes médicinales et aromatiques), 29 espèces et 61 variétés de fruits, 10 espèces et 15 variétés d'arbres et arbustes non fruitiers, et 75 espèces et 116 variétés de fleurs. Les jardins familiaux permettent d'ailleurs une production beaucoup plus importante et variée que des jardins partagés, à visée plus éducative que nourricière (voir ci-dessous un exemple de jardin en juin 2015 (fig. 13)). Il est également intéressant de noter qu'en fonction de leur origine, les jardiniers ne cultivent pas les mêmes produits, mais il arrive que des échanges de plants et de graines, ainsi que de fruits et légumes aient lieu. J'ai moi-même bénéficié, durant mon enquête, de la générosité des jardiniers toujours désireux de partager leurs productions. La plupart d'entre eux possèdent un congélateur qui leur permet de conserver les fruits et légumes afin de les consommer tout au long de l'année. Ils sont également nombreux à préparer des confitures, mais seul Marcel continue à faire des bocaux. L'autosuffisance vivrière n'est pas forcément le but de la majorité des jardiniers, même si certains d'entre eux sont près de l'atteindre.

Figure 13

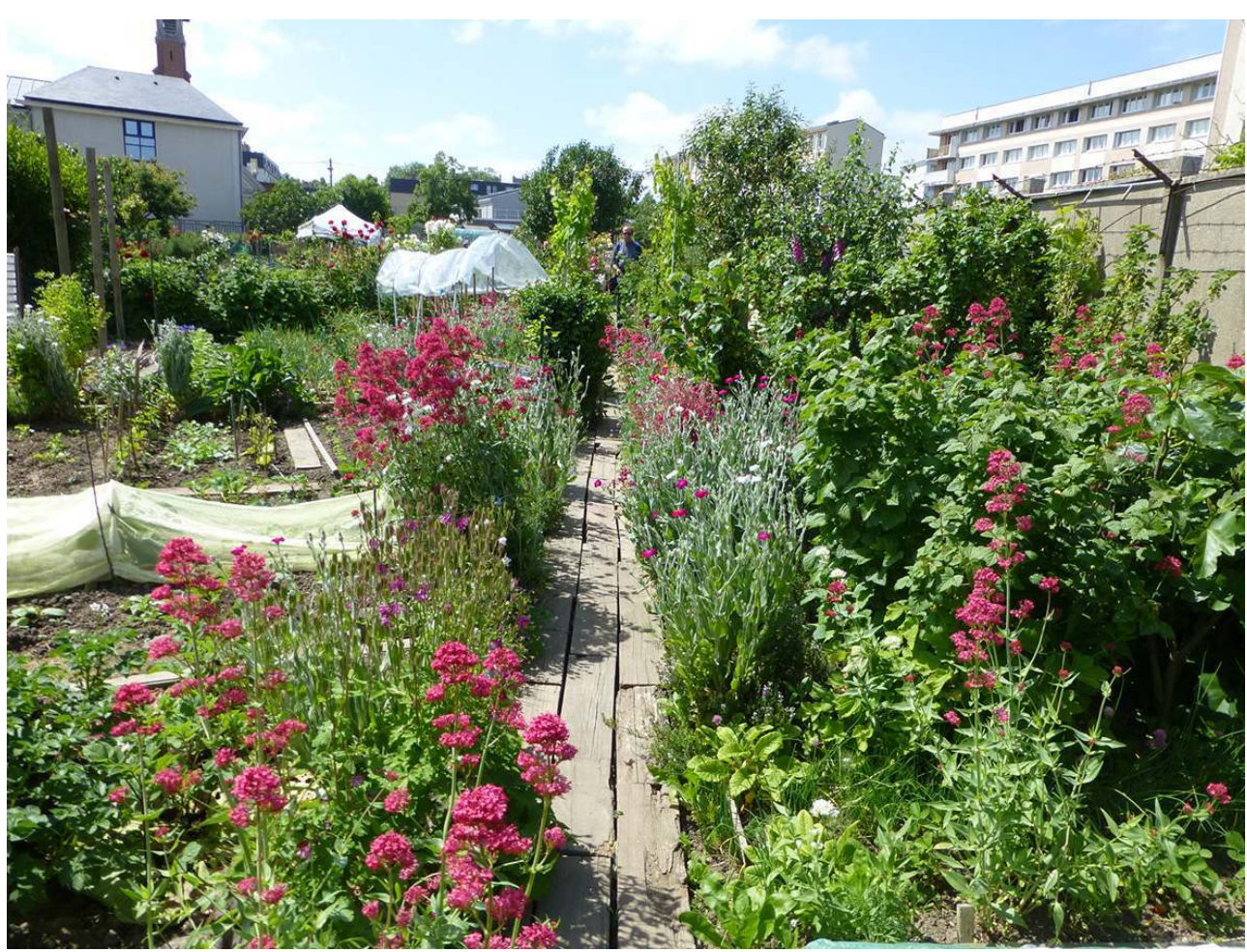

Le jardin de Teresa, jardins familiaux des Petits-Bois, Versailles, juin 2015.

(c) Fabienne Boursier. 


\section{Conclusion}

17 Cet article s'inscrit dans la continuité du combat mené par l'association gestionnaire et les jardiniers pour défendre l'existence des jardins familiaux des Petits-Bois. En effet, ces jardins ont été menacés à plusieurs reprises depuis leur création en 1906. La destruction de la maison du gardien en 2014 est la dernière concrétisation de ces menaces répétées. Autrefois situés dans un cadre rural, ils sont aujourd'hui des jardins urbains confrontés à la pression foncière. Leur inscription dans l'histoire sociale, horticole et urbaine locale et nationale leur confère un caractère patrimonial indéniable. Leur labellisation «jardin remarquable » fin 2014, du fait de leur caractère historique, ainsi que de leurs atouts vivriers, sociaux et environnementaux en milieu urbain, a été l'occasion de mener une enquête ethnographique sur le site auprès des jardiniers en 2015. La restitution des résultats de cette enquête permet d'obtenir une photographie sociale de la population des jardiniers et de décrire le site tel qu'il est aujourd'hui. Les témoignages prouvent que ces jardins familiaux rendent possible une certaine autonomie en matière de production vivrière et peuvent contribuer à terme à l'émergence d'une alternative à l'agriculture intensive et à l'agro-industrie. Ces jardins sont en outre le lieu de pratique et de transmission de savoir-faire jardiniers. Leur valorisation et leur sauvegarde sont donc primordiales, tant en raison de leurs vertus sociales, vivrières et environnementales (dans un quartier densément bâti et dans un contexte global de difficultés économiques et sociales grandissantes), qu'en tant que patrimoine populaire local et national.

\section{Textes juridiques}

Articles L564-1 à L564-3 du Code rural et de la pêche maritime. Titre VI : Jardins familiaux - Chapitre IV : Avantages et subventions (dernière modification : 12 mars 2018).

Circulaire n²004-003, du 17 février 2004, relative à la politique en faveur des jardins. Loi n52-895 du 26 juillet 1952, portant codification de la législation des jardins familiaux.

Loi n²014-366 du 24 mars 2014 (dernière modification : 29 janvier 2017), pour l'accès au logement et un urbanisme rénové (1) (Alur).

Webographie

Site internet officiel de l'Association des jardins familiaux de Versailles et des communes environnantes, créé en 2017, <http://lesjardinsfamiliauxdeversailles.fr/> [consulté le $15 / 09 / 2017]$.

Site internet partenaire des Jardins familiaux des Petits-Bois, créé en 2015, <http://jardinsfamiliaux.wixsite.com/site-petits-bois> [consulté le 15/07/2017].

\section{BIBLIOGRAPHIE}

AGGÉRI, Gaëlle. Inventer les villes-natures de demain : gestion différenciée, gestion durable des espaces verts. Dijon : Éducagri, 2010. 
BACHER, Rémi, LECLERC, Blaise. Une bonne terre pour un beau jardin : paillage, engrais verts, grelinette. Mens : Terre vivante, 2009.

BATAILlON, Agnès, [et al.] (dir). Jardins en banlieue. Paris : Créaphis, CAUE Val-de-Marne, 2003.

BERGUES, Martine. En son jardin : une ethnologie du fleurissement. Paris : Éd. de la Maison des sciences de l'homme, 2011.

BORTOLOTTO, Chiara. Le Patrimoine culturel immatériel, enjeux d'une nouvelle catégorie. Paris : Éd. de la Maison des sciences de l'homme, 2011.

BOURSIER, Fabienne. Jardins familiaux et jardiniers des Petits-Bois à Versailles : perception et appropriation du lieu [document électronique]. Mémoire de recherche de Master 2 Jardins historiques, patrimoine et paysage. Versailles : ENSAV, Université Paris-1, 2015. <http:// www.culturecommunication.gouv.fr/Thematiques/Patrimoine-ethnologique/Travaux-derecherche/Liste-des-travaux-finances/Travaux-finances/Liste-par-ordre-alphabetique-desnoms-des-auteurs/B> [consulté le 10/07/2017].

BOURSIER, Fabienne. "Jardins familiaux et jardiniers des Petits-Bois à Versailles : perception et appropriation du lieu ». Dans La Nature, le jardin et l'Homme : préserver et innover. Actes du colloque scientifique de la SNHF, Bordeaux, 19 mai 2017. Paris : SNHF, 2017, p. 35-40.

CABEDOCE, Béatrice, PIERSON, Philippe (dir.). Cent ans d'histoire des jardins ouvriers, 1896-1996. La Ligue française du coin de terre et du foyer. Grane : Créaphis, 1996.

CORBIN, Alain (dir.). L'Avènement des loisirs, 1850-1960. Paris : Aubier, 1995.

DEDIEU, Françoise. Pratiques populaires de temps libre dans les jardins ouvriers. Thèse de doctorat de sociologie. Saint-Denis : université Paris 8, 2007.

DESNOS, Pierre. Histoire des jardins ouvriers et familiaux de Versailles, 1901-2011. Versailles : ASRIEUPE, 2011.

DESNOS, Pierre. Des âmes près du corps, Petits-Bois Jussieu Picardie Pershing. Versailles : ASRIEUPE, 2014.

DUBOST, Françoise. Les Jardins ordinaires. Paris : L'Harmattan, 1997. (1 ${ }^{\text {re }}$ éd. : Côté jardins. Paris : éditions Scarabée et compagnie, 1984).

DUBOST, Françoise. Vert patrimoine : la constitution d'un nouveau domaine patrimonial. Paris : Éd. de la Maison des sciences de l'homme, 1994.

FOURNIER, Mauricette (dir.). Labellisation et mise en marque des territoires. Actes du colloque CERAMAC 34, Clermont-Ferrand, 8-10 novembre 2011. Clermont-Ferrand : Presses universitaires Blaise Pascal, 2015.

FOURNIER, Laurent Sébastien. « L'expérience de l'inventaire, une opportunité pour la recherche anthropologique », In Situ [En ligne], 33 | 2017, mis en ligne le 30 octobre 2017, consulté le 15 novembre 2018. URL : http://journals.openedition.org/insitu/15465 ; DOI : 10.4000/insitu.15465.

HALGAND, Nathalie, WEILL, Joëlle, BAUDELET, Laurence (dir.). « Du jardin ouvrier au jardin partagé : un rôle social et environnemental ». Bibliothèque numérique de l'INP, dossier documentaire préparé pour le séminaire de formation permanente de l'INP, Paris, 4-6 juillet 2007 [document électronique]. Paris : INP, 2007, 4, <http://mediatheque-numerique.inp.fr/var/ ezdemo_site/storage/original/application/bc70e0d918678d6d3ab253a476c1d1ce.pdf> [consulté le 20/07/2017].

HEINICH, Nathalie. "Synthèse et débat : un inventaire pour l'Europe? ». Dans Le Patrimoine culturel immatériel de l'Europe, inventer son inventaire. Comptes rendus de colloque [document 
électronique]. Paris : INP, 2009. <http://www.inp.fr/Mediatheque-numerique/Colloques/Lepatrimoine-culturel-immateriel-de-l-Europe-inventer-son-inventaire/Synthese-et-debats-Uninventaire-pour-l-Europe> [consulté le 15/07/2017].

HUBERT, Corinne. Fonds de l'association des jardins familiaux de Versailles et des communes environnantes (1903-1988). Répertoire numérique [...]. Versailles : Archives communales de la Ville de Versailles, 2012.

JACKSON, John Brinckerhoff. À la découverte du paysage vernaculaire. Trad. Xavier Carrère. Paris/ Versailles : Actes Sud/ENSP, 2003 (Éd. originale : Discovering the vernacular landscape. Yale : Yale University, 1984).

Les Jardins familiaux au cœur des villes. Actes du $34^{\mathrm{e}}$ congrès international des Jardins familiaux. Lyon : Fédération nationale des jardins familiaux et collectifs, 2006.

LENIAUD, Jean-Michel. L'Utopie française, essai sur le patrimoine. Paris : Mengès, 1992.

Mémento du jardin écologique. Versailles : Ville de Versailles, [s. d.].

NILSEN, Micheline. The Working man's green space : allotment gardens in England, France, and Germany, 1870-1919. Charlottesville : University of Virginia Press, 2014.

PASQUIER, Élisabeth, PETITEAU, Jean-Yves (collab.), CORIOU, Delphine (ill.), MERLET, Annabelle (ill.). Cultiver son jardin : chroniques des jardins de la Fournillère, 1992-2000. Paris : L'Harmattan, 2001.

PLUVINAGE, Manuel, WEBER, Florence. Les Jardins populaires : pratiques culturales, usages de l'espace, enjeux culturels. Éléments d'histoire et d'ethnographie. Rapport de recherche. Paris : Ministère de la Culture, Mission du patrimoine ethnologique, 1992.

QUELLIER, Florent. Une histoire du jardin potager. Paris : Armand Colin, 2012.

Versailles, ville verte, ville jardin. Versailles : Ville de Versailles, direction des Espaces verts, [s. d.].

TROCHET, Jean-René, PÉRU, Jean-Jacques, ROY, Jean-Michel (dir.). Jardinages en région parisienne du XVII ${ }^{e}$-XXe siècle. Grane : Créaphis, 2003.

Villes jardinées et initiatives citoyennes, Actes du colloque international, 11-12 octobre 2012.

Strasbourg: Ville de Strasbourg/AFDJEVP/JTSE/CNFPT, 2012.

WEBER, Florence. L'Honneur des jardiniers : les potagers dans la France du XX ${ }^{e}$ siècle. Paris : Belin, 1998.

\section{NOTES}

1. - BOURSIER, Fabienne. Jardins familiaux et jardiniers des Petits-Bois à Versailles: perception et appropriation du lieu. Mémoire de recherche de master 2 JHPP. Versailles: ENSAV, Université Paris-1, 2015.

2. - DESNOS, Pierre. Versailles, des âmes près du corps, Petits-Bois Jussieu Picardie Pershing. Versailles : ASRIEUPE, 2014, p. 10.

3. - DESNOS, Pierre. Histoire des jardins ouvriers et familiaux de Versailles, 1901-2011. Versailles: ASRIEUPE, 2011, p. 8.

4. - Ils deviendront jardins familiaux à partir de 1952, en vertu de la loi nº 52-895 du 26 juillet 1952.

5. - L'inscription des jardins ouvriers et familiaux dans l'histoire sociale est développée dans ce même numéro, dans le cas précis des Petits-Bois, par : DESNOS, Pierre. «Des jardins familiaux centenaires labellisés : quel avenir ? L'exemple du site Paul Philippe à Versailles ", In Situ [En ligne], 37 | 2018, mis en ligne le 12 décembre 2018, consulté le 19 décembre 2018. URL : http:// journals.openedition.org/insitu/18798, et plus généralement, par: DUBOST, Françoise. 
«Introduction générale », In Situ [En ligne], 37 | 2018, mis en ligne le 14 décembre 2018, consulté le 19 décembre 2018. URL : http://journals.openedition.org/insitu/19624.

6. - DESNOS, Pierre. Versailles, des âmes près du corps, Petits-Bois Jussieu Picardie Pershing. Versailles : ASRIEUPE, 2014, p. 4.

7. - Ancienne CEuvre des jardins ouvriers de Versailles et aujourd'hui Association des jardins familiaux de Versailles et des communes environnantes.

8. - DESNOS, Pierre. Histoire des jardins ouvriers et familiaux de Versailles, 1901-2011. Versailles : ASRIEUPE, 2011.

9. - Remplacé en 2017 par la Commission nationale du patrimoine et de l'architecture, «section parcs et jardins ».

10. - Ces aspects sont développés dans BOURSIER, F. Op. cit.

11. - Plan local d'urbanisme de Versailles (Projet d'aménagement et de développement durable (PADD), dossier approuvé en septembre 2006 - révisé en novembre 2011), p. 21.

12. - Loi n ${ }^{\circ}$ 2014-366 du 24 mars 2014 pour l'accès au logement et un urbanisme rénové (1).

13. - Ministère de la Cohésion et des Territoires. La Loi Alur: point d'étape, 23 mars 2016, voir le site: http://www.cohesion-territoires.gouv.fr/la-loi-alur-point-d-etape-1812 [consulté le 20/02/2018].

14. - FOURNIER, Mauricette (dir.). Labellisation et mise en marque des territoires. Actes du colloque CERAMAC 34, 8-10 novembre 2011. Clermont-Ferrand : Presses universitaires Blaise Pascal, 2015, p. 626-627.

15. - La retranscription de l'intégralité des entretiens se trouve dans BOURSIER, F. Op. cit., p. 202 à 380.

16. - DESNOS, P. Op. cit., p. 40.

17. - Selon les articles L564-1 à L564-3 du Code rural et de la pêche maritime.

18. - Le quatre-vingtième correspond à une parcelle inoccupée ou octroyée à une école primaire.

19. - DESNOS, P. Op. cit., p. 94.

20. - Selon leur corps de métier, les artisans sont considérés comme techniciens ou comme ouvriers.

21. - Mémento du jardin écologique. Versailles : Ville de Versailles, [s. d.].

22. - Ces produits, aux propriétés insecticides et fongicides naturelles, sont obtenus à partir des graines de margousier (neem). Bien qu'autorisés à la commercialisation par la Commission européenne depuis 2011, ils sont interdits d'utilisation en France, car aucune demande d'homologation en tant que produit phytosanitaire n'a été déposée.

23. - Le tableau détaillé des résultats se trouve dans BOURSIER, F. Op. cit., p. 170-178.

\section{RÉSUMÉS}

L'article s'attache à mieux faire connaître les jardins familiaux dits des Petits-Bois, à Versailles (Yvelines), à valoriser leurs atouts historiques, vivriers, sociaux et environnementaux, et à prévenir les menaces qui pèsent sur leur existence, au premier rang desquelles la pression foncière. Ces jardins s'inscrivent dans l'histoire horticole, sociale et urbaine locale et nationale, et le caractère patrimonial populaire qui leur est ainsi conféré justifie, entre autres raisons, l'attribution du label «jardin remarquable» par le ministère de la culture et de la Communication en 2014. L'auteur s'appuie sur les données de l'enquête ethnographique qu'elle a 
menée auprès des jardiniers des Petits-Bois en 2015, à la suite de cette labellisation. Elle décrit les caractéristiques du site actuel, présente la population des jardiniers et la diversité de leurs origines. Elle s'intéresse également à leurs pratiques jardinières: l'apprentissage et la transmission de savoir-faire, les produits utilisés, l'amendement de la terre et la rotation des cultures, ainsi que la variété des plantes cultivées. Elle aborde enfin les différends qui opposent les jardiniers entre eux.

This article is a presentation of the family allotment gardens known as the 'Petits-Bois' gardens, situated at Versailles near Paris in the Yvelines department. It sets out to describe their values in terms of historical, food provision, social and environmental considerations, and to warn of the dangers that threaten their existence today, primarily real estate pressures. The gardens are a part of local and national horticultural, social and urban history, and their popular appreciation as heritage led to their being awarded the French culture ministry's 'Remarkable Garden' label in 2014. The article is based on the results of an ethnographical research project carried out in 2015 on the Petits-Bois gardeners, after the garden had received this label. It describes the characteristics of the site today and presents the gardening population in all the diversity of its origins. It is also interested in specific gardening practices such as apprenticeship, the transmission of gardening skills, the products used, the improvement of the soils and the rotation of cultures and varieties of plants cultivated. In conclusion, it also looks at some of the points of disagreement that have arisen amongst the gardeners.

\section{INDEX}

Keywords : allotment garden, historical garden, 'Remarkable Garden' label, ethnographic heritage, intangible heritage, Versailles, subsistence farming, urban agriculture, gardening, social history

Mots-clés : jardins familiaux, jardins historiques, label « jardin remarquable », patrimoine ethnographique, patrimoine immatériel, Versailles, agriculture vivrière, agriculture urbaine, jardinage, histoire sociale

\section{AUTEUR}

\section{FABIENNE BOURSIER}

Historienne de l'art et des jardins, diplômée du Master 2 Jardins historiques, Patrimoine et Paysage (École nationale supérieure d'architecture de Versailles - Université Paris 1 PanthéonSorbonne) fabboursier@laposte.net 